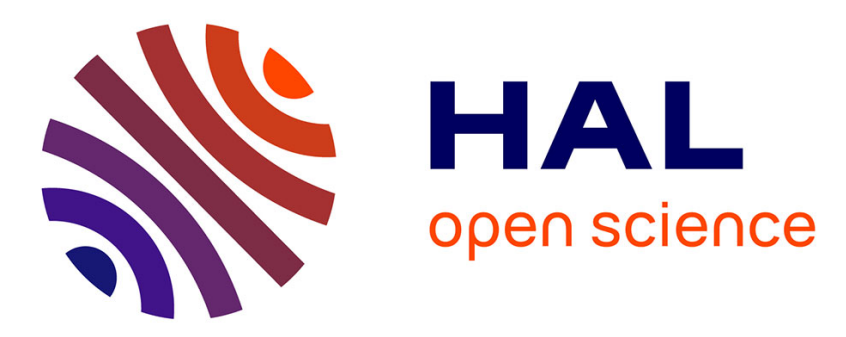

\title{
Internal Friction of Flux Motion in Hg-System High-Tc Superconductors
}

\author{
W. Tian, J. Zhu, H. Shao, J. Li, Y. Wang
}

\section{To cite this version:}

W. Tian, J. Zhu, H. Shao, J. Li, Y. Wang. Internal Friction of Flux Motion in Hg-System High-Tc Superconductors. Journal de Physique IV Proceedings, 1996, 06 (C8), pp.C8-497-C8-500. 10.1051/jp4:19968108 . jpa-00254536

\section{HAL Id: jpa-00254536 https://hal.science/jpa-00254536}

Submitted on 1 Jan 1996

HAL is a multi-disciplinary open access archive for the deposit and dissemination of scientific research documents, whether they are published or not. The documents may come from teaching and research institutions in France or abroad, or from public or private research centers.
L'archive ouverte pluridisciplinaire HAL, est destinée au dépôt et à la diffusion de documents scientifiques de niveau recherche, publiés ou non, émanant des établissements d'enseignement et de recherche français ou étrangers, des laboratoires publics ou privés. 


\title{
Internal Friction of Flux Motion in $\mathrm{Hg}-$ System High- $\boldsymbol{T}_{\mathrm{c}}$ Superconductors
}

\author{
W. Tian, J.S. Zhu, H.M. Shao, J. Li and Y.N. Wang \\ National Laboratory of Solid State Microstructures, Nanjing University, Nanjing 210093, China
}

\begin{abstract}
The internal friction(IF) and modulus as functions of temperature were measured for several Hg-system high-Tc superconductors(Hg1201, Hg1223, Hg1223 doped with $\mathrm{Fe}$ and $\mathrm{Pb}$ ), under the applied magnetic field, with vibrating reed technique. An IF peak associated with flux motion can be found below Tc for all samples. The temperature of the IF peak increases with reducing vibrating amplitude. This amplitude dependence of IF indicates that the flux motion is characterized by nonlinear behavior. No apparent shift of IF peak position can be detected by varying the frequency in the range from $10^{2} \mathrm{~Hz}$ to $10^{3} \mathrm{~Hz}$. Furthermore, the IF peak height satisfies a scaling law $\mathrm{Q}^{-1} \propto \omega^{-\mathrm{n}}$. This may be originated from phase transition of flux line lattice(FLL) rather than a thermally activated diffusion process.
\end{abstract}

\section{INTRODUCTION.}

Because of the great importance of flux pinning for achieving useful values of critical current in type-II superconductors, there has been continuing interest in investigating the vortex-pinning center interactions, as well as the collective properties of vortex assembly.

The behavior of flux line lattice(FLL) in superconductors has been studied extensively by measuring the magnetization, the susceptibility, the critical current and the mechanical response of the vortex. The first mechanical measurements developed were audio-frequency measurements using vibrating reeds[1] and high$\mathrm{Q}$ mechanical oscillators[2]. This technique involves measuring the resonance frequency and dissipation in an oscillator made of a superconductor, or which has a superconductor attached. A feature common to all the mechanical measurements of flux motion in superconductors materials is that a dissipation peak and a coriesponding decrease in the stiffness of FLL is seen below Tc when the temperature is swept at constant magnetic field. There is currently a controversy as to the origin of this feature. While some authors consider it to be evidence for flux lattice melting[2;3] or some transition to a glassy state of the flux structure[4], others interpret the measurements in terms of thermally activated flux diffusion[5].

The difference between these two mechanisms concentrates on whether the flux motion is controlled by a thermally activated process or not. For the diffusion which depends on the thermally activated process, the temperature of IF peak may 
decrease with reducing the measuring frequency. However, for the "transition" process, the position of IF peak is frequency independent. Therefore, detecting the frequency dependence of the IF peak temperature becomes crucial for determining the actual mechanism of the flux dissipation.

In this paper we report on mechanical measurement of dissipation associated with the flux motion by vibrating reed technique in $\mathrm{Hg}$-system superconductors.

e find no apparent shift of IF peak position in the frequency range from $10^{2}$ to $10^{3} \mathrm{~Hz}$. However, the scaling law of IF peak height $\mathrm{Q}^{-1} \propto \omega^{-n}$ is first discovered in our measureinents. This may be originated from melting phase transition of FFL rather than a thermally activated diffusion process.

\section{EXPERIMENTS:}

The $\mathrm{HgBa}_{2} \mathrm{CuO}_{8+y}\left(\mathrm{Hg}_{1201}\right), \quad \mathrm{HgBa}_{2} \mathrm{Ca}_{2} \mathrm{Cu}_{3} \mathrm{O}_{8+y}\left(\mathrm{Hg}_{1223}\right)$, ( $\left.\mathrm{Hg}_{0.66} \mathrm{~Pb}_{0.34}\right) \mathrm{Ba}_{2} \mathrm{Ca}_{2} \mathrm{Cu}_{3} \mathrm{O}_{8+y}(\mathrm{Hg} 1223-\mathrm{Pb}), \mathrm{HgBa}_{2} \mathrm{Ca}_{2}\left(\mathrm{Cu}_{0.98} \mathrm{Fe}_{0.02}\right)_{3} \mathrm{O}_{8+\mathrm{y}}$ ( $\mathrm{Hg} 1223-$ Fe) were synthesized following the procedure described else where[6]. The critical temperature is $96 \mathrm{~K}, 120 \mathrm{~K}, 135 \mathrm{~K}, 116 \mathrm{~K}$ for $\mathrm{Hg} 1201, \mathrm{Hg} 1223, \mathrm{Hg} 1223-\mathrm{Pb}$, $\mathrm{Hg} 1223-\mathrm{Fe}$, respectively. The X-ray analysis shows these samples are nearly single-phase polycrystalline. The vibrating reed consists of a platelet clamped at one end, while two electrodes near the end serve to detect its motion electrostatically. The platelet is a Si crystals sputtered with a metal film, with the superconducting sample glued to it. The magnetic field is transverse field.

\section{RESULTS and DISCUSSION}

Fig1. displays the $\mathrm{Q}^{-1}$ data as a function of temperature for different applied fields for $\mathrm{Hg} 1223$. Similar results are also obtained in all samples.

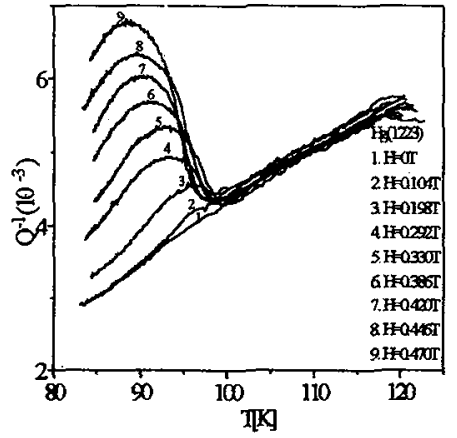

Figl. The temperature dependence of $Q^{-1}$ at different magnetic fields

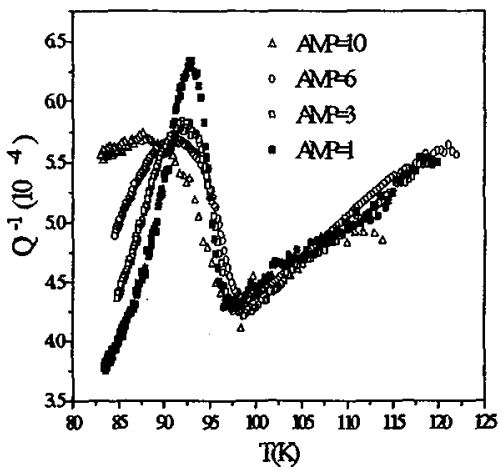

Fig2. The temperature dependence of $\mathbf{Q}^{-1}$ at different amplitudes

Fig2. shows the temperature of the response of $Q^{-1}$ in different vibrating amplitude for $\mathrm{Hg} 1223$. Similar results are also found in $\mathrm{Hg} 1223-\mathrm{Pb}$. It can be 
seen that the IF peak shifts towards lower temperature with increasing vibrating amplitude, which shows the nonlinear effect characterizing the flux motion.

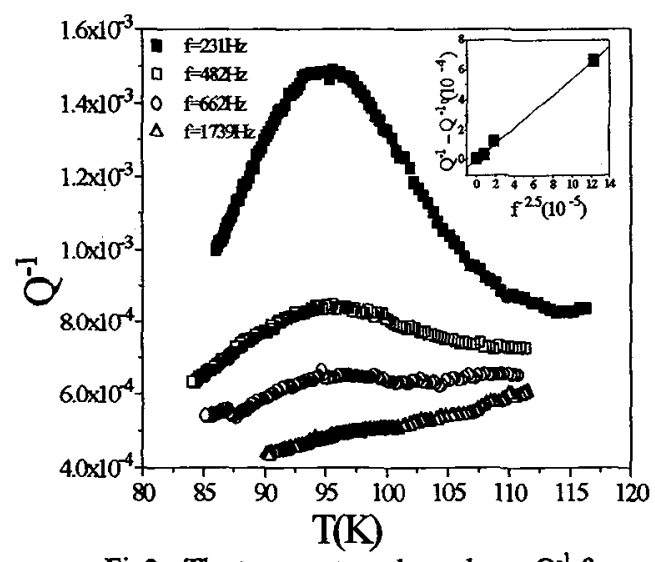

Fig3. The temperature dependence $Q^{-1}$ for different frequency

Fig3. is our main result. The sample was $\mathrm{Hg} 1223-\mathrm{Pb}$. Simliar results were obtained in $\mathrm{Hg} 1223$ and $\mathrm{Hg} 1223-\mathrm{Fe}$. As displayed in the Fig3., the IF peak position does not shift with varying the frequency in the range $10^{2} \leq \omega \leq 10^{3} \mathrm{~Hz}$ and the IF approximately satisfies the scaling law $\mathrm{Q}^{-1} \propto \omega^{-2.5}$, which indicates the character of a phase transition rather than a thermally activated diffusion of flux. The frequency dependence of the dissipation of vibrating superconductor at constant temperature and DC magnetic field can be used to test the mode of the FLL dynamics. However, it is impossible to achieve the vibrating-reed measurements in the frequency window that spans several decades. Gupta et al [7] fabricated vibrating reeds with the same thickness $d p$ but different lengths $l p$. The resonance frequency of these reeds varies according $\omega \propto d p / l p^{2}$. Thus one expects a frequency-independent dissipation since only the product $\omega l p^{2}$ enters the expression for the dissipation. But in our measurements, we regulate the vibrating frequency by varying the length of Si host with the superconducting sample's length unchanged. Therefore, the frequency-dependent IF peak position should be found if the flux motion is a thermally activated diffusion.

Our results show that the IF peak position is nearly frequency independent and the IF peak height is obviously frequency dependent, which can not be interpreted in terms of thermal activated flux diffusion. Up to now, many experiments indicated a melting process occurs at a temperature lower than $\mathrm{Tc}[8]$. Due to our experimental results, we offer the conjecture that the microscopic origin of the dissipation is the motion of the interface boundary between the FLL liquid phase and FLL lattice phase. The dissipation peak emerges as a direct consequence of the fact that the density of interface boundaries is only appreciable in the immediate vicinity of the melting point. The $\mathrm{Q}^{-1} \propto \omega^{-n}$ relation was also seen elsewhere[9], in other experimental results associated with the first order phase transitions in solids. Zhang $e t$ al. presented a theoretical model to explain the change in $\mathrm{Q}^{-1}$ in solids arising from the reconstruction and displacement of crystal structure and motion of the phase interface[10]. We considered the $Q^{-1}$ change in our measurements has the same origin. The further studies are under performing. 


\section{Acknowledgments:}

We are grateful to Dr. H.L.Zhou, H.F.Wang, Z.Yang for their help with the measurements. This work was supported by National Research and Development Center on Superconductivity in Beijin.

\section{References}

[1] Brandt E.H., Esquinazi P. , and Neckel H. , J.Low.Temp.Phys. 63 (1986) 187216. Esquinazi P. Neckel H. , Weiss G., and Brandt E.H., J.Low.Temp.Phys. 64 (1986) 1-20.

[2] Gammel P.L. , Schneemeyer L.F. , Wasczak J.V. , and Bishop D.J., Phys.Rev.Lett. 61 (1988) 1666-1669.

[3] Nelson D.R. , Phys.Rev.Lett. 60 (1988) 1973. Fisher M.P.A. , Phys.Rev.Lett. 62 (1989) 1415-1418.

[4] Gregory S. , Rogers C.T. , Venkatesan T. , Wu X.D. , Inam A. , and Dutta B. , Phys.Rev.Lett. 62 (1989) 1548-1551.

[5] Ziese M. , Esquinazi P. and Braun H.F. , Supercond.Sci.Technol. 7 (1994) 869-890.

[6] Shao H.M. , Zhou L.J., Shen J.C. , Hua X.Y. , Yuan P.F. , Yao X.X. , Physica C 232 (1994) 5-13.

[7] Gupta A. , Esquinazi P., Braun H.F., Gerhauser W. , Neumuller W.H. , Heine K. and Tenbrink J. , Europhys.Lett. 10 (1989) 663-668.

[8]Safar H. , Gammel P.L. , Huse D.A. , Bishop D.J. , Rice J.P. , and Ginsgerg D.M. , Phys.Rev.Lett. 69 (1992) 824-827.

[9] Wang Y.N. et al J.Phys. (Paris) Colloq. 42 (1981) C5-1049. Zhang J.X. , Zheng W. , Fung P.C.W. , and Liang K.F. , J.Alloys compounds. 211/212 (1994) 378.

[10] Zhang J.X. , Fung P.C.W. , and Zeng W.Z. ., Phys.Rev. B 52 (1995) 268277. 